den alterthiimlichen Namen Kyanos angedeutet, in welcher Beziehung also, dem nächsten $Z$ wecke der vorliegenden Zeitschrift gemäss, selbst aus dem alten $\mathrm{H}$ o $\mathrm{m}$ er etwas zu lernen für praktische Chemie.

\title{
XLIII.
}

\section{Fortsetzung der Untersuchung des Platinrückstandes, nebst vorläufiger Ankündigung eines neuen Metalles. Vom}

Prof. C. Claus*).

(Bulletin scient. de St. Petersbourg.)

Die Mittheilung des Herrn F rémy (Comptes rendus XVIII, p. 144-147**)) über die Darstellung des Osmiums und Iridiums aus dem Platinrückstande veranlasst mich, meine fortgesetzten, noch nicht vollendeten Arbeiten über diesen Gegenstand vorläufig bekannt zu machen.

Ich bin zu ähnlichen Resultaten als Herr Fr e m y gelangt, jedoch auf einem anderen Wege, der mich zur Auflindung eines neuen Metalles, das ich Ruthenium zu nennen gedenke, führte.

Ich gehe nun zur Erörterung meiner Untersuchungen über. Es wurden 15 Pfund Rückstand auf einmal in Arbeit genommen, und dieser nicht, wie bei meinen frühern Untersuchungen, mit Chlor aufgeschlossen, weil diese Methode, ungeachtet ihrer grossen Vorzuige, beim Arbeiten im Grossen umständlich und schwierig ist, sondern ich schmolz ihn mit Salpeter, und zwar je 1 Pfund Rückstand mit 2 Pfund dieses Salzes in einem geräumigen hessischen Tiegel, bei starker Glühhitze 2 Stunden hindurch. Die geschmolzene Masse wurde in eine eiserne Schale ausgegossen und bei der weiteren Bearbeitung derselben ein von dem gewöhnlichen Verfahren abweichender, mehr complicirter Weg eingeschlagen, weil das von mir schon früher bemerkte neue Metall aufzusuchen war. Die Masse wurde nämlich mit Wasser ausgelaugt, und

*) Dies. Journ. Bd. XXXII. S. 479. - Die vorläufige Ankïndignng des Rutheniums ist im Journ. Bd. XXXIV. S. 173 gegeben.

**) Dies. Journ. Bd. XXXIII. S. 407. 
die ganze Arbeit zerfiel nun in zwei Hauptoperationen, in die Bearbeitung der wässrigen Lösung $\boldsymbol{A}$ und des in Wasser unlöslichen Theiles $B$.

\section{A. Bearbeitung der Lösung.}

Diese ist nach dem Abstehen und Klären braunroth, riecht etwas nach Osmiumsäure und enthält (der Menge nach in folgender Reihe) chromsaures, osmiumsaures, kieselsaures Kali, iridiumsaures, rutheniumsaures und titansaures Kali, ferner salpetrigsaures Kali und freies Kali. (Das rutheniumsaure Kali hielt ich bei dieser ersten Arbeit für iridiumsaures Kali.) Setzt man etwas Salpeteroder Schwefelsäure hinzu, so wird viel Osmiumsäure frei und es fällt ein grünlich-schwarzer voluminöser Niederschlag heraus, der Osmiumoxyd-, Iridiumoxyd- und Rutheniumoxyd-Kali, Chromsäure Kieselsäure und Titansäure enthält. Da ich bedeutende Quantitäten, wohl ein paar 100 Litres hatte, so dampfte ich sie, olne zuvor Säure hinzugesetzt zu haben, in einem grossen eisernen Kessel bis zu einem geringen Volumen ein. Hierbei setzte sich ein bedeutender schwarzgrüner Niederschlag ab, welcher dem durch Säuren erhaltenen ähnlich war. Die Operation zerfällt nun abermals in zwei Theile, in Bearbeitung des Niederschlages $a$ ) und der Flüssigkeit $b$ ).

a) Der Niederschlag bestand aus einem feinen, voluminösen schwarzgrünen Pulver und kleinen granatrothen Octaëdern. Diese liessen sich durch Sieden mit Wasser herausziehen und krystallisirten beim Erkalten der Lösung. Beim langsamen Erkalten bekam ich ziemlich grosse schwarze glänzende Krystalle; schneller erkaltet, schossen kleine granatrothe 0ctaëder an, und beim Umrühren der in kaltes Wasser gestellten Lösung mit einem Glasstabe sonderte sich ein rosenrothes krystallinisches Pulver ab. Die verschiedenartig gefärbten Krystalle hatten ein gleiches Verhalten; fein zerrieben, gaben sie ein weisses, in's Röthliche schielendes Pulver, hatten einen süsslich zusammenziehenden Geschmack und lösten sich sehr langsam und schwer in kaltem Wasser mit der Farbe des mangansauren Kali's. In siedendem Wasser sind sie leicht löslich, allein es zerlegt sich ein Theil in Osmiumsäure und schwarzes 0smiumoxyd. Eine vollständige Zersetzung erfolgt angenblicklich, wenn man zu der Lösung etwas $\mathrm{SO}_{3}$ oder $\mathrm{N}_{5}$ setzt, wobei eine grosse Menge Osmiumsäure frei wird und sich ein sammetschwarzer, voluminöser Niederschlag absondert. Dieses 
Oxyd schwindet sehr beim Trocknen und stellt so eine zusammengebackene trockene Substanz von dunkelumbrabrauner Farbe dar, welche einen schwachen metallischen Schimmer hat. Es ist ein Hydrat und zerfällt beim Erhitzen in einer Glasröhre in wässerige Osmiumsäure und metallisch glänzendes 0smium. Es löst sich leicht in Salzsäure mit dunkelbraun-grüner Farbe; Salmiak bringt in dieser Lösung einen geringen Niederschlag eines braunen krystallinischen Salzes (wahrscheinlich OsmiumsesquichlorürChlorammonium), der grösste Theil bleibt gelöst mit dunkelgrüner Farbe. Die Lösıng des 0xyds in Salzsäure giebt beim Abdampfen grünes lösliches Osmiumchlorür. Das Oxyd behält hartnäckig einen Antheil der Säure, mit welcher gefällt worden, zurück. Leitet man über das mit Salpetersäure gefällte 0xyd beim Glühen Wasserstoff, so bleibt eine messinggelbe metallisch glänzende Substanz zuriuck, wahrscheinlich Osmiumstickstoff. Die Versuche, dieses Oxyd zu analysiren, misslangen alle, weil dasselbe beim Erhitzen mit Hydrogen schwach verpufft und umhergeschleudert wird.

Das Verhalten des granatrothen Osmiumsalzes brachte mich auf die Vermuthung, dass es eine Verbindung einer noch unbekannten Oxydationsstufe des Osmiums mit Kali sei, und zwar KO $+\mathrm{Os}_{3}$; allein die sehr genau übereinstimmenden Resultate von melıreren Analysen kamen der Zusammensetzung des osmiumsauren Kali's sehr nahe, so dass ich verleitet wurde, es für dieses, bisher noch nicht dargestellte Salz zu halten. Ich erhielt nämlich bei der Reduction des Salzes mittelst Wasserstoffgas und beim Auffangen des gebildeten Wassers immer 4 Atome Wasser auf 1 Atom Metall und 1 Atom Kalihydrat; es musste also die Formel folgende sein : $\mathrm{KO}, \mathrm{Os}_{4}+\mathrm{Aq}_{q}^{*}$ ). In dieser Meinung wurde ich

*) Die Analyse des osmigsauren Kali's ist etwas schwierig und giebt stets einen bedeutenden Verlust an Osmium, weil bei dem Trocknen ein Theil des Osmiums als Osmiumsäure entweicht und bei der Reduction mit Hydrogen ein geringer Theil unreducirt bleibt, selbst bei der Rothrlihhitze. Das Salz wurde nach dem Trocknen bei $100^{\circ} \mathrm{C}$, in einem Schiffchen von reinem Silber abgewogen, in eine Glasröbre gethan, deren Gewicht genau bestimmt war, diese wie bei einer organischen Analyse mit einem Chlorcalciumrecipienten versehen und in mit Magnesia ausgefütterten Blechschienen im Lieb i g'schen Ofen geglüht, wäbrend Wasserstuff hindurchgeleitet wurde. Nach dem Versuche wurde die Chlorcalciumröhre und die andere, das redacirte Salz enthaltende Röbre gewogen und so die Menge des gebildeten Wassers und des Verlustes der Substanz bestimmt. Die schwarze Masse in dem Silbersehiffchen wurde mit Wasser ausgelangt. Die Lösung enthielt 
noch mehr bestärkt, als ich mir das Salz direct aus 0smiumsäure und überschüssiger Kalilösung beim Abdampfen in einer Retorte

noch etwas unzersetztes osmigsaures Kali, die Menge war aber böchst unbedeutend. Sie wurde bei einem Zusatze von Schwefelsäure bis zur Trockne abgeraucht, geglïht und das Kali als schwefelsaures Kali bestimmt. Das Metall wurde auf einesn gewogenen Filter gesammelt, gut ausgewaschen, bei $100^{\circ} \mathrm{C}$. getrocknet und gewogen. Auf gleiche Weise analysirte ich das Barvtsalz. Die Analyse gab folgende Resultate:

I. 1,315 Grm. gaben :

$$
\text { Osmigsaures Kali. }
$$

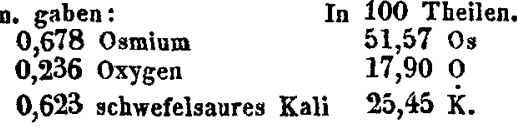

II. 1,272 Grm. Eaben:

$\begin{array}{ll}0,657 \text { Os } & 51,80 \text { Os } \\ 0,220 \text { o } \ldots & 17,29 \text { o } \\ 0,617 \text { K } \dddot{\mathbf{S}} & 26,18 \text { K. }\end{array}$

III. 1,299 Grm. gaben:

\begin{tabular}{|c|c|}
\hline 0,672 os & $\begin{array}{l}51,68 \\
17,90\end{array}$ \\
\hline $0,630 \dot{\mathrm{K}} \ddot{\mathbf{S}}$ & 26,18 . \\
\hline
\end{tabular}

IV. $1,320 \mathrm{Grm}$. gaben :

$\begin{array}{ll}\text { rm. gaben: } & \\ 0,680 \text { os } & 51,65 \\ 0,228 \text { o } \ldots . . & 17,00 \\ 0,625 \text { K } \mathbf{s} & 25,45 .\end{array}$

V. 1,982 Grm. gaben:

$\begin{array}{ll}1,024 \text { Os } & 51,65 \\ 0,339 \text { o } \ldots & 17,15 \\ 0,940 \text { K } \dddot{s} & 25,63 .\end{array}$

Die Formel $\mathrm{KO}, \mathrm{Os}_{4}+1 \mathrm{Aq}$ fordert nach der Berechnung:

$$
\begin{array}{cl}
53,02 & \mathrm{O}_{3} \\
17,11 & \mathrm{O} \\
25,13 \mathrm{KO} \\
0,71 \mathrm{Aq}
\end{array}
$$

Die Formel $\mathrm{KO}, \mathrm{Os}_{3}+2 \mathrm{Aq}$ erfordert 52,54 Osmium.

I. 1,065 Grm. gaben:

$$
\begin{aligned}
& \text { gaben: } \\
& 0,409 \text { os } \\
& 0,165 \text { o } \\
& 0,603 \text { Ba }
\end{aligned}
$$$$
\text { Osmigsaurer Baryt. }
$$

In 100 Theilen. 46,95 os 15,520 37,15 Ba.

II. 0,830 Grm. zaben :

$$
\begin{array}{ll}
0,386 \text { os } & 46,54 \text { Os } \\
0,128 \text { o } \ldots & 15,40 \text { o } \\
0,472 \text { Ba } \mathrm{s} & 37,25 \mathrm{Ba} .
\end{array}
$$

Die Formel $\mathrm{BaO}, \mathrm{OsO}_{3}+\mathrm{H}_{2} \mathrm{O}$ erfordert:

$$
\begin{array}{ll}
47,61 & \text { Os } \\
15,30 & 0 \\
36,61 & \text { Ba } \\
0,48 & \text { H. }
\end{array}
$$


darstellte. Es war hier keine Reduction vorauszusetzen. Nachdem mir aber Frém y's Arbeit zu Gesichte gekommen war und ich seine Versuche wiederhoite, wurde ich von meinem Irrthume überzeugt und das Salz muss die Formel KO, Os $\mathrm{O}_{3}+2 \mathrm{Aq}$ haben. Ich bemerkte nun, dass sich die Osmiumsäure mit Kali beim Erhitzen ohne Concurrenz eines Desoxydationsmittels zu $\mathrm{Os}_{3} \mathrm{O}_{3}$ reducirt. Uebersättigt man nämlich flüssige Osmiumsäure mit Kali und thut Säure hinzu, so erfolgt keine Ausscheidung von Osmiumoxyd. Erhitzt man aber die Flüssigkeit einige Zeit, so färbt sie sich braunroth, und nun erfolgt bei Süurezusatz ein reichlicher schwarzer Niederschlag. Diese Desoxydation der Osmiumsäure bei Gegenwart von Kali ist sehr merkwürdig, da dem gerade entgegen alle Oxyde des Osmiums, auch selbst Metall, an der Luft sich höher oxydiren und Osmiumsäure ausdünsten. Osmigsauren Baryt erhält man, wenn man eine heisse Barytlösung zu flüssiger Osmiumsüure giesst und in einer verschlossenen Flasche lüngere Zeit stehen lüsst. Anfangs bildet sich ein grüner flockiger, formloser Niederschlag, welcher nach und nach in sternförmige Krystalldrusen, die aus schwarzen diamantglänzenden Prismen bestehen, sich umwandelt. Das Salz ist unlöslich in Wasser. Es gab bei der Analyse 1 Atom Baryt, 1 Atom Metall und 4 Atome Wasser. Die Formel ist also $\mathrm{Ba} \mathrm{O}$. $\mathrm{Os}_{3}+\mathrm{HO}$. - Die Lösung des osmigsauren Kali's giebt mit Metallsalzlösungen schwarze oder braune Niederschläge.

Das von dem osmigsauren'Kali befreite schwarze Präcipitat $a$ ) wurde in einer Retorte mit Königswasser übergossen und der Destillation unterworfen, wobei viel Osmiumsäure überging. Der his zur Trockne abdestillirte Retorteninhult wurde mit Wasser und Salzsäure ausgezogen; er hinterliess unreine, titansäurehaltige Kieselsäure. Zur Lösung wurde viel Salpetersäure gethan und nochmals destillirt, dieses Mal aber nur Spuren von Osmiumsäure gewonnen. Der Retorteninhalt wurde mit vielem Chlorkalium vermischt, wobei ein Salz niederfiel, das ich damals für KaliumIridium-Chlorid hielt, welches aber bedeutende Antheile von Rutheniumsalz enthalten haben musste, wie mich spätere Untersuchungen über das Ruthenium belehrt haben. Leider habe ich das Salz einem bedeutenden Vorrathe von Iridiumsalz beigegeben. Die von dem Iridium-Doppelchloride abfiltrirte Flüssigkeit hatte 
eine tiefgrüne Farbe und gab mit Ammoniak viel unreines kieselhaltiges Chromoxydhydrat.

b) Diese sehr concentrirte gelbbraune Flüssigkeit wurde in einer Retorte so lange mit verdünnter Schwefelsäure vermischt, bis sie schwach sauer reagirte. Es entwickelte sich hierbei unter starker Effervescenz Kohlensäure, salpetrige Säure und Osmiumsäure wurde frei. Bei der Destillation ging sehr reine Osmiumsäure über, welche sich in sehr schönen weissen Krystallen in der Vorlage sammelte. Die Destillation wurde so lange fortgesetzt, bis keine 0 smiumsäure mehr überging. In der Retorte blieb eine gelbe Flüssigkeit, welche chromsaures Kali enthielt, und ein schwarzes Präcipitat, dem Präcipitate $a$ ) in der Zusammensetzung gleich, mit Ausnahme des Gehaltes an osmigsaurem Kali. Es wurde wie der Niederschlag $a$ ) behandelt.

Die bei dieser und späteren Bearbeitungen des Platinrückstandes gewonnene Osmiumsäure wurde aus ihren Lösungen mit H S als Schwefelosmium gefällt und dieses als Hauptmaterial zur Darstellung der meisten Osmiumverbindungen benutzt. Schwefelosmium mit gleichen Gewichtstheilen Chlorkalium gemischt und in einer grossen Porcellanröhre mit feuchtem Chlorgase behandelt, giebt halium-0smiumchlorid, welches mit metallischem 0smium gemengt in der Röhre bleibt, während Chlorschwefel und etwas 0 smiumsäure in das vorgeschlagene Wasser übergehen. Dieses färbt sich nach einiger Zeit dunkelblau und enthält die blaue Verbindung von Osmium und schwefliger Säure. Kali fält aus dieser Lösung ein blaues Oxyd. - Laugt man den Inhalt der Röhre mit siedendem Wasser aus, so bleibt eine grosse Ifenge metallisches Osmium zurück und aus der Lösung krystallisirt beim Erkalten und nachmaligen Abdampfen das 0smiumsalz in schönen braunrothen Octaëdern. Aus der Mutterlauge fällt man mit kohlensaurem Natron beim Erwärmen schwarzes 0smiumoxyd. So habe ich durch eine einfache Operation 3 Osmiumverbindungen und me tallisches Osmium gewonnen; nämlich eine Unze 0smium, 2 Unzen Kalium-0smiumchlorid, 2 Drachmen Osmiumoxyd 0 s $\mathrm{O}_{2}$ und $1 / 2$ Drachme blaues 0smitumoxyd. Auch kann man sich aus dem Schwefelosmium die Säure darstellen, wenn man dasselbe in einer Röhre, beim Glühen, mit Sauerstoff behandelt und die Producte in eine kleine stark abgekühlte Vorlage auffängt. Die 0smiumsäure geht in flüssiger Form über und erstarrt in dem Recipienten, zu- 
gleich sammelt sich darin eine dunkelblane Flüssigkeit, die schon erwähnte Verbindung der Osmiumsäure mit $\mathrm{S} \mathrm{O}_{2}$; diese höchst flüchtige, für die Gesundheit sehr gefährliche Verbindung lässt sich leicht von der reinen Osmiumsäure abgiessen. Diese Darstellungsweise ist jedoch zur Gewinnung der Osmiumsäure nicht vortheilhaft, weil viel Säure in die blaue Verbindung eingeht.

\section{B. Bearbeitung des in Wasser unlöslichen Theiles der mit Salpeter geschmolzenen Masse.}

Je 2 Pfund desselben wurden mit 1 Pfund Künigswasser und der gehörigen Menge destillirten Wassers in einer Retorte der Destillation unterworfen, bis der Inhalt fast trocken war. Das Destillat enthielt viel Osmiumsäure. Die Masse in der Retorte wurde mit siedendem Wasser aufgeweicht und in grosse Zuckergläser zum Abklären gegossen, und später von dem Bodensatze $c$ ) decantirt, dieser noch mit Wasser bis zur Erschöpfung ausgelaugt. Ich vermischte nun die Flüssigkeit bis zur alkalischen Reaction mit Potaschenlösung und erhielt einen starken Niederschlag von gelbbraunem Eisenoxydhydrat, den ich mehrere Tage in der Flüssigkeit liess, wobei er sich schwarzbraun färbte. Diese Färbung glaubte ich einem Antheile mit niedergefallenen Iridiumoxyds zuschreiben zu können, vermuthete aber auch darin etwas Rhodiumoxyd. Ich sammelte daher das unreine Eisenoxyd, löste es in Salzsäure und erhielt eine tief purpurrothe, fast schwarze, undurchsichtige Lösung. Diese Erscheinung war mir sehr auffallend, da keins der mir bekannten Oxyde sich mit einer solchen Farbe in Säure löst. Aus dieser Lösung erhielt ich durch's Fällen mit Zink ein Metall, das sich weder wie Rhodium, noch wie Iridium verhielt. Es gab nämlich, mit Kochsalz gemischt und mit Chlor in der Glühhitze behandelt, eine schwarze Masse, welche sich in Wasser mit pomeranzengelber Farbe löste; diese Lösung, deren Farbe sehr leicht von den Lösungen des Rhodiums und Iridiums, so wie eines Gemenges beider Metalllüsungen, unterschieden werden konnte, gab mit Ammoniak einen sammetschwarzen Niederschlag und färbte sich, mit $H S$ behandelt, unter Abscheidung eines schwarzen Schwefelmetalles intensiv lasurblau. Weder Iridium noch Rhodium zeigt ein ähnliches Verhalten. Chlorkalium und Ammonium geben mit der Lösung dieses Metalles schwerlöslich krystallinische Salze, die nur schwer von den Doppelchloriden des 
Iridiums zu unterscheiden sind, wenn man sie nicht unter einem guten Mikroskop betrachtet. Diese grosse Aehnlichkeit verleitete mich anfangs, das Metall für unreines Iridium zu halten, dessen ungewoohnliche Reactionen von einem mir unbekannten Körper abhängig sein mochten. Ich suchte nun diesen hörper von dem Iridium zu trennen, allein meine Mühe war vergeblich. Ich fing nun an, diesen Körper in allen möglichen Beziehungen mit dem Iridium zu vergleichen, und fand dabei so grosse Verschiedenheiten, dass mir ïber seine Eigenthümlichkeit kein Zweifel übrig blieb. Bei dieser Gelegenheit wurde ich mit seinen Eigenschaften so vertraut, dass es mir leicht wurde, eine sichere Methode aufzufinden, ihn aus dem Platinrückstande darzustellen und von den andern Metallen zu trennen. Ich hatte bis jetzt nur sehr wenig davon erhalten, weil es aus der Lösung des unreinen Eisenoxyds weder durch Zink noch Schwefelhydrogen vollkommen gefält wird. Später fand ich noch eine namhafte Mènge desselben in dem unlöslichen Räckstande $c$ ). Ich werde später wieder auf dieses Metall zurückkommen.

Die Lösung, aus der das Eisenoxyd mit Kalilösung gefältt worden war, hatte in den hölzernen Gefässen, in welchen sie längere Zeit gestanden hatte, fast gänzlich ihre braune Farbe eingebüsst, sie war fast farblos, nur in's Gelbliche spielend. Vermischte man sie mit einer sehr gesättigten Potaschenlösung, so entstand ein weisser Niederschlag, der sich bräunte, grau oder rosenroth wurde, beim Trocknen aber wieder seine weisse Farbe annahm. Dieser Körper hatte alle Eigenschaften des von O sann (Poggendorff's Annalen, Bd. XIV. S. 329) beschriebenen Kutheniumoxyds und ich habe mich von der Identität beider Körper überzeugt. Salzsäure zog ein Metalloxyd mit grünlicher Farbe aus, das, mit Salpetersäure gekocht, eine pomeranzengelbe Auflösung gab, die sich mit Schwefelhydrogen blau färbte. Es enthielt also das neue Metall. Das Ungelöste bestand grösstentheils aus titanhaltiger Kieselsäure, Thonerde und wahrscheinlich auch Zirkonerde. Auch 0 sann hate sein rohes Rutheniumoxyd, um es zu reinigen, mehrere Male mit Salzsäure behandelt, allein diese Lösung nicht untersucht; so ist ihm die Entdeckung entgangen. Weil dieses Metall in dem Osann'schen Rutheniumoxyde in geringer Menge vorkommt, wünsche ich es Ruthenium zu nennen; sollte aber im Verfolge meiner 
Untersuchung sich ergeben, dass mein Ruthenium das Pluran Osann's ist, so könnte der von mir gewählte Name doch bleiben, weil 0 sann anfangs die Absicht hatte, das Pluran Ruthenium zu nennen. Es ist leicht möglich, dass das Pluran Osann's ein höheres Oxyd oder Chlorid meines Rutheniums ist; leider wissen wir aber von dem Pluran fast nichts. Es ist flüchtig und giebt in der weissen Verbindung mit $\mathrm{HS}$ ein graues Schwefelmetall. Die oben angeführte L'ösung, von der ich an 150 Litres hatte, wurde nur zum Theil zur Gewinnung des Rutheniums benutzt, reil man auf diese Weise, bei einer sehr unangenehmen Arbeit, sehr wenig gewinnt und die Darstellung der übrigen Metalle sehr erschwert wird. Der grösste Theil wurde in einem eisernen Kessel bis zu einem geringen Volumen eingeraucht; hierbei schied sich viel blaues Iridiumoxyd $a b$, das von der Flüssigkeit, welche salpetersaures Kali und Chlorkalium enthielt, durch's Abseihen getrennt wurde. Das blaue 0xyd löste ich in Königswasser, rauchṭ bis zu einem geringen Volumen ein und vermischte die Lösung mit Chlorkalium, wobei Kalium-Iridiumchlorid gewonnen wurde, das durch öfteres Umkrystallisiren in grossen, schönen Octaëdern gewonnen wurde. Es war vollkommen rein. Die Mutterlauge des Iridiumsalzes wurde bis zur Trockne abgeraucht und mit Hydrogen reducirt. Das gewonnene Metallpulver enthielt Iridinm-Ruthenium und sehr wenig Rhodinm. Aus demselben stellte ich mir, auf die später zu beschreibende Weise, durch's Schmelzen mit Salpeter Ruthenium dar.

Auf diese Weise waren die durch die erste Schmelzung mit Salpeter aufgeschlossenen Substanzen getrennt und dargestellt worden.

C. Bearbeitung des nach der ersten Schmelzung und Ausziehung mit Wasser und Säuren zurïckgebliebenen Restes.

Er betrug 11 $\frac{1}{2}$ Pfund und stellte ein feines, schwarzes Pulver dar, in welchem keine metallisch glänzenden Flitter von OsmiumIridium zu bemerken waren. Er war fast frei von Osmium, enthielt aber noch viel Iridium, Chromeisenstein, Silicate, Ruthenium und Rhodium. Dieses letztere Metall suchte ich besonders in diesem Reste auf, da es bisher in kaum bemerkbarer Spur vorgekommen war, während ich beim Aufschliessen mit Chlor bedentende Antheile desselben gewonnen hatte. Ich fing meine 
Proben im Kleinen an, indem ich eine Portion dieses Restes mit Chlor aufschloss, die Lösung mit einer siedenden; concentrirten Boraxlösung vermischte und den sich hierbei abscheidenden braunen Niederschlag, der grösstentheils aus borsaurem Eisenoxyde bestand, in Salzsäure löste und aus dieser das Netall mit Zink fällte. Ein. Theil des erhaltenen Metalles wurde mit Chlor und Kochsalz aufgeschlossen, die Masse in Wasser gelöst, fast bis zur Trockne abgeraucht und dann mit Alkohol ibergossen. Dieser löste Natrinm-Rutheniumchlorid und liess Natrium-Rhodium zurück. Der andere Theil des Metalles wurde mit Salpeter geschmolzen und hierauf mit Wasser ausgelaugt, es blieb Rhodium zurück, rutheniumsaures Kali wurde gelöst. Eine andere Portion des Restes wurde ein paar Mal mit Salpeter geschmolzen und mit Wasser ausgelaugt. Der ungelöste Theil wurde mit Chlor aufgeschlossen, die Lösung mit Borax gefällt. Hierbei entstand kein brauner, sondern ein gelber Niederschlag, welcher aus borsaurem Eisenoxyde und Rhodiumoxyde bestand. Die Auflösung dieses Niederschlages in Salzsäure war nicht wie früher, wo sie Ruthenium enthielt, schwarz purpurfarben, sondern hellgelb und gab, mit Zink gefällt, chemisch-reines Rhodium, das keine Spur Iridium und Ruthenium enthielt. Diese und frühere Versuche beweisen, 1) dass das Ruthenium von schmelzendem Salpeter in rutheniumsaures Kali verwandelt wird, das sich in Wasser vollkommen löst; 2) dass die purpurrothe Farbe des unreinen Eisenoxyds von der Gegenwart des Rutheniumoxyds abhängig ist; 3) dass das Rhodium und Ruthenium vom Borax sehr leicht gefäll werden, das Iridium aber nur unter gewissen Bedingungen; 4) dass das mit Salpeter geglühte Rhodium nur sehr wenig in Säuren löslich ist; 5) dass das Natrium-Rutheniumehlorid in Alkohol löslich ist.

Es war mir nun leicht, das in dem Reste c) vorhandene Ruthenium auf eine einfache Weise zu gewinnen. Es wurde der ganze Rest abermals mit Salpeter geglüht, jedoch nur gleiche Theile beider Körper genommen, und zwei Stunden lang in einem hessischen Tiegel der Weissgliuhhitze ausgesetzt. Die breiartige Masse wurde noch heiss mit einem eisernen Spatel herausgenommen. Nach dem Erkalten ühergoss man sie mit destillirtem Wasser (anderes Wasser taugt nicht dazu) und lässt die Flüssigkeit durch's Abstehen sich kJüren; man decantirt vorsichtig ab, giesst 
frisches Wasser zu und laugt auf diese Weise so lange aus, bis nichts mehr gelöst wird. Die schön pomeranzengelbe Lösung enthält chromsaures, rutheniumsaures und kieselsaures Kali, keine Spur Iridium. Thut man nur sehr wenig Salpetersäure hinzu, so entsteht ein voluminǒser, sammetschwarzer Niederschlag, der unreines, kieselsäurehaltiges Rutheniumoxyd-Kali ist. Dieses übergiesst man mit Salzsäure, worin es sich anfangs mit grüner, hierauf beim Erhitzen mit schöner pomeranzenrother Farbe löst. Die Lösung raucht man fast bis zur Trockne ein, wobei man aber Sorge tragen muss, dass immer freie Säure vorhanden ist. Beim Verdünnen mit vielem Wasser fällt die Kieselsäure gallertartig heraus. Man filtrirt die Lösung, engt sie bis zu einem geringen Volumen ein und vermischt sie mit einer gesättigten Lösung des Chlorkaliums. Hierbei fällt Kalium-Rutheniumchlorid heraus. Die Lauge giebt beim Abdampfen noch viel Salz. Dieses reinigt man durch Umkrystallisiren. Dieses Salz ist das Material, aus dem man sich das Metall und seine noch wenig untersuchten Verbindungen darstellen kann.

Weiter bin ich mit der Untersuchung des Platinrückstandes nicht gekommen; mit dem Auffinden des Rutheniums blieb die ganze Arbeit liegen und ich beschäftige mich gegenwärtig nur mit analytischen Untersuchungen seiner Verbindungsverhältnisse, wobei seine Eigenthümlichkeit noch evidenter wird.

Ich kann gegenwärtig nur wenig von diesem Körper mittheilen, da mir der Gegenstand noch zu neu ist.

Das Metall ist pulverförmig schwarzgrau, wenig glänzend, wenn es aus dem Chloridsalze reducirt wird, metallisch glänzend, weissgrau, wenn man es aus dem $0 x y d e \mathrm{Ru}_{2} \mathrm{O}_{3}$ durch Reduction gewinnt, wahrscheinlich sehr schwer schmelzbar. Es ist bedeutend leichter als Iridium. 0,838 Grm. desselben nehmen bei 2 stündigem Glühen 0,155 Grm. Sauerstoff auf, indem es in ein blanschwarzes Oxyd verwandelt wird, das in der Weissglühitze nicht reducirt wird. Wasserstoffgas reducirt es beim Glühen. Beim Schmelzen mit saurem schwefelsaurem Kali wird es nicht gelöst. Schmilzt man es jedoch mit Salpeter, so erhält man eine schwarzgrüne Masse, welche sich in Wasser mit pomeranzengelber Farbe zu rutheniumsaurem Kali löst, das einen schwachen, aber ganz eigenthümlichen Geruch hat. Säuren fällen ein schwarzes Ruthenium- 
oxyd heraus. Aetzkali löst das Metall beim Glïhen eben so leicht auf als Salpeter.

Schon diese Eigenschaften sind hinreichend, das Ruthenium von dem Iridium und Rhodium zu unterscheiden, allein es hat deren noch mehrere. Besonders ausgezeichnet sind die Reactionen der Lösung seines Chlorids gegen gewisse Substanzen. Schwefelhydrogen z. B. fällt das Metall nur zum Theil als schwarzes Sulfuret, während das Chlorid in ein schönes lasurblaues Chlorür übergeführt wird, welches von Schwefelhydrogen sehr schwer zersetzt wird.

Thut man in die Lösung des pomeranzengelben Chlorids eine Zinkstange, so färbt sich die Flüssigkeit nach einiger Zeit ebenfalls lasurblau, sie entfärbt sich aber später und es wird metallisches Ruthenium gefält, jedoch nicht ganz vollständig.

Alkalien und Borax fällen das Chlorid mit schwarzer Farbe, indem alkalihaltiges $0 x y d$ herausfällt.

Kaliumeisencyanür verändert die Lösung anfangs nicht, später tritt Entfärbung ein und zuletzt nimmt dieselbe eine chromgrüne Farbe an.

Quecksilbercyanür bewirkt einen schwarzblauen Niederschlag und blaue Färbung der Lösung.

Eins der vorzüglichsten Reagentien für dieses Metall und das Iridium zugleich ist das salpetersaure Silberoxyd. Es fällt nämlich die Lösung des Rutheniumchlorids mit schwarzer Farbe. Der Niederschlag ist ein Gemenge von Chlorsilber und Rutheniumoxyd; er löst sich nach 24 Stunden zum Theil auf, indem das Rutheniumoxyd (wahrscheinlich unter Sauerstoffverlust) sich in der freien Salpetersäure mit kirschrother Farbe löst, während das Chlorsilber mit weisser Farbe zurückbleibt. Thut man zu dieser Flüssigkeit Ammoniak im Ueberschusse, so löst sich das Chlorsilber und schwarzes Rutheniumoxyd fallt heraus. Eben so verhält sich das Iridiumchlorid, nur unter anderen Erscheinungen. Es erfolgt beim Zusatze des salpetersauren Silberoxyds ein indigoblauer Niederschlag, ein Gemenge von Iridiumoxyd und Chlorsilber; dieser fängt jedoch schon nach einigen Augenblicken an sich zu entfärben und nach zwei Stunden ist die Entfärbung vollständig, auf dem schwach gelblich gefärbten Chlorsilber ist die Flïssigkeit farblos. Thut man hierauf Ammoniak hinzu, so löst sich das Chlorsilber und das Iridium bleibt als eine gelblich- 
weisse Verbindung ungelöst zurück*). Dieses Reagens ist für das Iridium so fein, dass die geringste Spur desselben in Gemengen von Lösungen anderer Metalle entdeckt werden kann.

Das Metall bildet unter gewissen Bedingungen Chloride von den verschiedenen Farben des Regenbogens, grüne, blaue, violette, purpurrothe, kirschrothe und pomeranzengelbe. Das letztere ist wahrscheinlich das höchste Chlorid und hat die Zusammensetzung $\mathrm{Ru}_{2} \mathrm{Cl}_{3}$; es ist das beständigste und lässt sich leicht auf die früher angegebene Weise darstellen, besonders als Doppelsalz des Kaliums und Ammoniums. Ersteres $=2 \mathrm{~K} \mathrm{Cl}+\mathrm{Ru}_{2} \mathrm{Cl}_{3}$, bildet sehr kleine glänzende Krystalle, welche $\frac{1}{10} \sigma$ Linie im Durchmesser haben und deren Form bei einer 400 maligen Vergrösserung deutlich wahrgenommen werden kann. Es sind pomeranzengelbe, durchsichtige Cuben, in denen die 0ctaëderform vorgezeichnet ist, mit ganz merkwürdigen Zeichnungen in den Richtungen der Axen; oft kommen darin aber auch Krystalle anderer. Form und Farbe vor, welche anderen Chlorstufen anzugehören scheinen. Ohne Vergrösserung erscheint das Salz als ein braunes, in's Violette spielendes, krystallinisches Pulver. Das neutrale Salz zersetzt sich in der wässerigen Lösung sehr leicht, besonders beim Erwärmen, die Lösung wird dunkler, zuletzt ganz schwarz und undurchsichtig, und es setzt sich daraus ein schwarzer, voluminöser Niederschlag $a b$, wahrscheinlich eine basische Verbindung, während die Flüssigkeit noch trübe und dunkelgefärbt erscheint. Bei Gegenwart freier Säıre erfolgt diese Zersetzung nicht. In diesem zersetzten Zustande hat das Salz eine ungemein stark tingirende Kraft, welche abhängig ist von der grossen Zertheilung des schwarzen Niederschlages, welcher sich zum Theil schwebend in der Flüssigkeit erhält und diese tingirt. Löst man nämlich ein Milligramm Salz in 4 Grm. Wasser und erhitzt die schwach gelbgefärbte Flüssigkeit, so wird sie ganz schwarz wie Tinte und kann noch mit $30 \mathrm{Grm}$. Wasser vermischt

*) Dieses Verbalten scheint mir für die Geschichte des Iridiums von nicht geringer Wichtigkeit; es dentet auf die Wahrscheinlichkeit, dass das blaue Iridiumoxyd, so wie ich schon früher in meiner letzten Abhandlung angedeutet habe, Ir $\mathrm{O}_{2}$ ist, eine der beständigsten und bestimmtesten Oxydationsstufen des Iridiums; ferner, dass dieses Oxyd eine Säure ist, welche sich nicht mit den Sauerstoffsäuren obne Verlust von Oxygen verbinden kann. Der Verfolg einer genanen Untersuchung dieser Reaction wird, wie ich hoffe, bedeutende Aufschlüsse über die Oxydationsstufen des Iridiums geben. 
werden, ohne bedeutend an Intensität abzunehmen. Das Salz enthält nur 28,90 Metall, also tingirt ein Theil desselben mehr als 100,000 Theile Wasser, fast mit der Farbe einer blassen Tinte, die jedoch etwas in's Braune schielt. Fällt man eine Lösung des $2 \mathrm{~K} \mathrm{Cl}+\mathrm{Ru}_{2} \mathrm{Cl}_{3}$ mit Ammoniak, so erhält man einen schwarzen Niederschlag, welcher Chlor und Ammoniak enthält und, in Salzsäure gelöst, hierauf bis zur Trockne abgeraucht, eine schmuziggrüne Salzmasse zurücklässt. Löst man diese in Wasser, so crhält man eine undurchsichtige, schwarzkirschrothe Flüssigkeit, welche bei einem geringen Zusatze von Salpetersäure und beim Abrauchen verschiedene Farben annimmt, und zwar Violett, Blau, Purpurroth, Kirschroth, Braun und Pomeranzengelb. - Das Salz ist ziemlich schwerlöslich in kaltem Wasser, leichter löslich in siedendem Wasser, unlöslich im Weingeiste. Es hat einen rein zusammenziehenden, nicht metallisch bittern Geschmack, während das Iridiumchlorid ekelhaft bitter schmeckt. Das Salz zu Pulver gerieben, nimmt eine gelbbraune Farbe an.

Das $\mathrm{Ru}_{2} \mathrm{Cl}_{3}$ erhält man, wenn man das reducirte Metallpulver mit Königswasser erhitzt. Ein kleiner Theil davon löst sich mit pomeranzengelber Farbe. Raucht man die Lösung bis zur Trockne ab, so bleibt eine gelbbraune, unkrystallisirte Masse zurïck, welche Feuchtigkeit anzieht und zerfliesst. Sie löst sich leicht in Wasser unter Zurücklassung einer gelbbraunen, basischen Verbindung.

Aus dem Kalium-Rutheniumchloride kann das Metall nicht durch's Glühen mit Natron reducirt werden, da hierbei sich das Ruthenium oxydirt und mit dem Alkali rutheniumsaures hali bildet, das sich in Wasser mit pomeranzengelber Farbe löst.

Leitet man über schwachgliihendes Ruthenium trockenes Chlorgas, so verflüchtigt sich ein Chlorid, das als gelber Rauch zur Röhre herausströmt. Der grösste Theil des Metalles bleibt jedoch unangegriffen zurück.

Es folgen nun einige vorläufige analytische Untersuchungen, welche hinreichend beweisen, dass dieses Metall kein Iridium ist.

Die Analyse der Hauptverbindung des Salzes $2 \mathrm{~K} \mathrm{Cl}+\mathrm{Ku}_{2}$ $\mathrm{Cl}_{3}$ wurde nach $\mathrm{zwei}$ sich gegenseitig controlirenden Methoden bewerkstelligt. Es wurde in einer Röhrenkugel abgewogen und mit Wasserstoff reducirt, der Gewichtsverlust bestimmt und als Chlor in Rechnung genommen. Das Gemenge von Chlorkalium Journ. f. prakt. Chemie. XXxiv. ?. 
und Metall wurde mit Wasser ausgelaugt, die Lösung in einem Platinschälchen abgeraucht und das trockene Salz schwach gegluht und gewogen. Das auf einem sehr kleinen Filter gesammelte Metall wurde in einen Platintiegel gethan, das Filter zu Asche verbrannt, dem Metalle hinzugefügt und in einem Strome von Wasserstoffgas geglüht.

Die andere Methode, welche die directe Bestimmung des Chlors zum Hauptzwecke hatte, bestand darin, dass man das in einem Platintiegel abgewogene Salz, mit der gehörigen Menge Aetzbaryt gemischt, mit Wasser anfeuchtete, eintrocknen liess und hierauf in einem Strome Wasserstoff reducirte. Die reducirte Masse aufgeweicht, wurde auf ein Filter gegeben, anfangs mit siedendem Wasser gut ausgelaugt, später mit salpetersäurehaltigem Wasser. Aus der Flüssigkeit wurde das Chlor auf die gewöhnliche Weise als Chlorsilber gefällt und dieses nach dem Schmelzen gewogen; ferner das Kali als schwefelsaures Kali bestimmt, indem man erst das Silber durch Salzsäure, dann den Baryt als schwefelsaures Salz entfernte und die Flüssigkeit bis zur Trockne abrauchte, das Salz glühte. Das Metall wurde wie früher bestimmt.

Die Darstellung eines zur Analyse tauglichen Salzes von bestimmter Zusammensetzung unterliegt vielen Schwierigkeiten, welche in der leichten Zersetzbarkeit des Rutheniumchlorids ihren Grund haben. Raucht man nämlich die Auflösung des Rutheniumoxyd-Kalf in Königswasser, mit Chlorkalium vermischt, ab, so krystallisirt ein Salz heraus, das nach dem Auswaschen und Trocknen zwar ein gleichförmiges, krystallinisches Pulver darstellt, an welchem man mit der Loupe keinen fremdartigen Körper entdeckt; betrachtet man es aber unter einer 400 fachen Vergrösserung, so nimmt man Krystalle von drei verschiedenen Formen wahr. Der grösste Theil besteht aus orangefarbenen, durchsichtigen Cuben, weniger häufig sind gelbe, durchsichtige Octaëder mit ganz eigenthümlichen Zeichnungen im Innern der Krystalle, ferner eine geringe Menge dunkelblauer Prismen und undurchsichtiger amorpher Körnchen. Die drei Krystallformen gehören wahrscheinlich drei verschiedenen Chlorstufen des Rutheniums an; der amorphe Körper ist ein basisches Chlorid des Rutheniums, welches sich beim Auswaschen des neutralen Salzes mit Wasser bildet. Um das cubische Salz in möglichster Reinheit zu gewin- 
nen, übergiesst man das gemengte Salz in einem Becherglase mit Wasser, welches mit Salzsüure sauer gemacht worden. Hierbei löst sich das octaëdrische und prismatische Salz, und das basische Pulver schlämmt sich in der Flüssigkeit auf, während das cubische Salz als eine feste Schicht sich am Boden des Glases ablagert, von dem die Lösung mit dem aufgeschlämmten Pulver leicht abgegossen werden kann. Man spült die Krystalle ein paar Mal mit etwas saurem Wasser ab und trocknet sie bei gewöhnlicher Temperatur. Ein so gereinigtes Salz zeigt unter dem Mikroskop nur cubische Krystalle ohne fremde Beimengung. Um es zur Analyse gehörig zu trocknen, wurde es bei $200^{\circ} \mathrm{C}$. mit Chlorgas behandelt.

I. 1,096 Grm. dieses Salzes gaben :

In 100 Theilen :

0,316 Metall 28,96 Ru

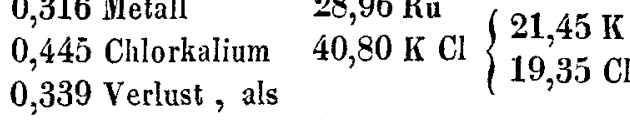

Chlor berechnet $30,24 \mathrm{Cl}$.

II. 0,990 Grm. dieses Salzes gaben:

In 100 Theilen :

$\begin{array}{ll}0,282 \text { Metall } & 28,48 \mathrm{Cl} \\ 0,409 \text { Chlorkalium } & 41,39-\left\{\begin{array}{l}21,71 \mathrm{~K} \\ 19,60 \mathrm{Cl}\end{array}\right\} 49,78 \frac{\mathrm{O}}{\mathrm{O}} \mathrm{Cl} . \\ \mathbf{0 , 3 1 0} \text { Verlust, als } \\ \quad \text { Chlor berechnet } 30,22-\end{array}$

III. 1,003 Grm. dieses Salzes gaben :

In 100 Theilen :

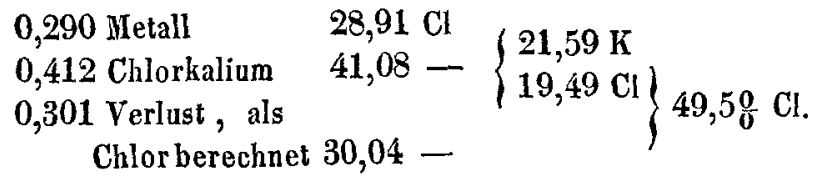

Directe Bestimmung des Chlors.

1. 0,704 Grm. gaben 1,364 Chlorsilber, also $48,30 \frac{0}{0}$ Chlor.

II. $0,654-1,268-18,95 \frac{0}{0}-$

Es war also noch etwas Wasser in dem Salze, doch weniger

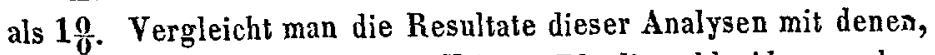
welche die Untersuchung des Kalium-Rhodiumchlorids gegeben haben (s. Berzelius in Poggendorff's Annalen, B. XIII. 
1828. S. 442), so ergiebt sich eine merkwürdige Uebereinstimmung; diese wird aber noch grösser, wenn man die Zusammensetzung der 0xyde des Rutheniums mit denen des Rhodiums vergleicht. $\quad 0,838 \mathrm{Grm}$. des pulverförmigen reducirten Rutheniums oxydirten sich anfangs beim Erhitzen sehr leicht und nahmen in kurzer Zeit 150 Oxygen auf, dann erfolgte die Oxydation langsamer. Nachdem beim Glühen auf der Weingeistlampe mit doppeltem Luftzuge das 0xyd nicht mehr am Gewichte zunahm, wurde es in einem Windofen einer heftigen Gluhhitze ausgesetzt, wobei noch einige Milligramme $0 x y g e n$ absorbirt wurden. Die ganze Menge des verschluckten $0 x y g e n s$ betrug $0,155 \mathrm{Grm}$., also auf 100 Theile Metall 18, 4 Oxygen.

0,752 Grm. eines schwarzen Ruthenium-0xydhydrats, das Jurch kohlensaures Kali aus der Lösung des Kutheniumchlorids gefält und darauf acht Tage hindurch ausgewaschen worden war, gaben bei der Analyse:

$$
\begin{aligned}
& \text { 0,166 Grm. Wasser, } \\
& \text { 0,102 0xygen, } \\
& \text { 0,424 Metall, } \\
& \text { 0,060 Kalihydrat. }
\end{aligned}
$$

Das wasserleere, kalifreie Oxyd enthält also auf 75,9 Theile Metall 18 Theile Sauerstoff, nahe so, wie Berzelius die Zusammensetzung des Rhodiumoxyds $\dddot{\dddot{*}}$ gefunden hat.

Es ist also das analysirte trockene Salz $=2 \mathrm{~K} \mathrm{Cl}+\mathrm{Ru}_{2} \mathrm{Cl}_{3}$, das durch's Glühen erhaltene $0 x y d=3 \dot{R} u+\dddot{u} u$, das gefällte Oxyd in reinem Zustande $\dddot{*} u$.

Hieraus ergiebt sich, dass das Ruthenium ein merkwürdiges Paarling des Rhodiums ist, das in seinen Verbindungsverhältnissen dem Rhodium ganz gleich ist. Man kann also mit grosser Wahrscheinlichkeit annehmen, dass es mit dem Rhodium isomorph ist und ein ihm gleiches Atomgewicht, nämlich 651,387 hat.

Die grosse Uebereinstimmung der analytischen Resultate konnte vermuthen lassen, dass das von mir aufgestelite neue $\mathrm{Me}$ tall Rhodium sei; allein die anderweitige grosse Verschiedenheit des Verhaltens beider Metalle, welche ich hier besonders hervorheben will, ist dieser Meinung entgegen.

1) Das Ruthenium, mit Salpeter geschmolzen, löst sich mit orangegelber Farbe vollständig in Wasser auf; eben so beim 
Schmelzen mit Kali; das Rhodium giebt bei ähnlicher Behandlungsweise ein braungrünes $0 x y d$, das weder in Wasser noch Säuren löslich ist.

2) Das Ruthenium wird beim Schmelzen mit saurem schwefelsaurem Kali nicht gelöst.

3) Das Rutheniumchlorid hat eine pomeranzengelbe Farbe und giebt, mit Alkalien behandelt, ein schwarzes 0xydhydrat. Das reine Rhodiumchlorid hat eine rosenrothe Farbe und giebt mit Alkalien ein hellgelbes $0 x y d$.

4) Schwefelhydrogen, durch eine Lösung des Rutheniumchlorids geleitet, verwandelt dieses unter Abscheidung von Schwefelruthenium in ein dunkelblanes Chlorür*); Rhodiumchlorid hingegen wird theils zersetzt, theils bleibt es mit rosenrother Farbe unzersetzt in der Lösung.

5) Die Lösung des neutralen Rutheniumchlorids zersetzt sich ungemein leicht, besonders beim Erwärmen, indem sie in Folge der Bildung einer basischen Verbindung schwarz und undurchsichtig wird. Das Rutheniumchlorid ist sehr beständig.

Schliesslich gebe ich noch ein Verfahren an, wie man sich durch einen einfachen Versuch von der Eigenthümlichkeit des Rutheniums überzeugen kann. Man braucht hierzu nur ein paar Milligramme des Metalles oder irgend einer seiner Verbindungen. Man schmilzt es nämlich mit einem grossen Ueberschuss von Sal-

*) Dieses blaue Chlorïr habe ich bisher nur in flüssiger Form erhalten können und zwar auf folgende Weise: Kalium-Ruthenitmchlorid wird in Wasser, dem etwas Salzsäure zugesetzt worden, beim Erwärmen gelöst und hierauf so lange mit $\mathbf{H}$ S behandelt, bis die Flüssigkeit indigoblau geworden ist. Hierauf filtrirt man sie von dem schwarzen $S$ chwefelruthenium ab and leitet, zur Entfernung des darin enthaltenen freien Gases, einen raschen Strom Luft hindurch, bis jeder Gertuch nach Schwefeluydrogen entfernt ist. Diese prächtig lasurblave, in dicken Schichten fast undurchsichtige Flüssigkeit ist bei gewöhnlicher Temperatur ziemlich beständig; sie giebt mit Ammoniak einen violettblauen Niederschlag, der aber nach einiger Zeit grau wird; die Flïssigkeit hat dann eine schmuzig gelbe Farbe. Raucht man die blaue Chlorürlösung ab, so nimmt sie bei starker Concentration eine schöne chromgrüne Farbe an, so dass die Flüssigkeit von Chromeblorïr nicht zu unterscheiden ist. Ammoniak giebt mit dieser einen dunkelgrüen Niederschlag, welcher sich beim Erbitzen mit der Flüssigkeit zum Theil mit kirschrother Farbe wieder auflöst, zum Theil in ein schwarzes, unlósliclres Oxyd übergeftibrt wird. Sowohl das blaue als das grüne Chloriir verwandeln sich in das orangegelbe Chlorid, wenn sie mit Salpetersäure erbitzt werden. Ist das blaue Chlorïr etwas verdïnnt und nit viel Salzsäure vermischt, so wird es beim Erhitzen farblos. Auch schweflige Säure bewirkt vollständige Entfärbung. 
peter in einem Platinlöffelchen bei starker Hitze so lange, bis die glühende Masse nicht mehr aufschäumt, sondern ruhig fliesst; dann lässt man erkalten und löst in wenigem destillirtem Wasser. Ein paar Tropfen Salpetersäure bringen in dieser pomeranzengelben Lösung einen voluminösen schwarzen Niederschlag als Rutheniumoxyd-Kali hervor. Thut man zur Flüssigkeit mit dem Niederschlage Salzsäure und erhitzt sie in einem Porcellanschälchen, so löst sich das Oxyd auf und nimmt bei der Concentration eine schöne orangegelbe Farbe an. Leitet man nun H S durch die Lösung, bis sie fast schwarz geworden ist, und filtrirt, so läuft eine Flüssigkeit von prachtvoll lasurblauer Farbe durch.

\title{
XLIV.
}

Ueber die chemische Zusammensetzung der Puzzolane und der vulcanischen Bomben.

\author{
Von \\ Dr. I. Elsner.
}

Ich habe im vorigen Jahre in diesem Journale, Band XXXIII. Heft 1. S. 21, die Analyse des rheinischen Cements mitgetheilt und a. a. 0 . angezeigt, dass ich später eine nach demselben Grundsatze ausgeführte Analyse der Puzzołane unternehmen lassen würde; diese Untersuchung ist mit Puzzolanen vom Vesuv, welche ich der freundlichen Mittheilung des Herrn K r a n z verdankte, im Laboratorium des königl. Gewerb-Instituts unter meiner Leitung von den Züglingen $R$ e in h ard $t$ und S te $n$ gel angestellt worlen; aus den Resultaten dieser Analysen, welche ich sogleich ausführlicher angeben werde, hat sich ergeben, wie schon im voraus anzunehmen war, dass auch die Puzzolane aus theils in Salzsäure aufschliessbaren, theils in dieser Säure nicht aufschliessbaren Silicaten bestehen, dass daher die Berthi er'sche Analyse derselben in dieser Hinsicht zu berichtigen ist. - Dass die Resultate der verschiedenen Analysen mit einander in quantitativer Beziehung nicht übereinstimmen können, ist wohl an sich klar, da diese Mineralien nur Gemenge sind; dass diese letzteren jedoch aus Silicaten von verschiedenem Ver- 